\title{
Pharmacology of new oral anticoagulants: mechanism of action, pharmacokinetics, pharmacodynamics
}

\author{
Luca Masotti, ${ }^{1}$ Mauro Campanini ${ }^{2}$ \\ ${ }^{1}$ Internal Medicine, Cecina Hospital, Italy; ${ }^{2}$ Internal Medicine, Novara Hospital, Italy
}

\begin{abstract}
Due to their mechanism of action, the new oral anticoagulants are named direct oral anticoagulants (DOACs). Dabigatran is a selective, competitive, direct inhibitor of thrombin (Factor IIa) while rivaroxaban, apixaban and edoxaban act by directly inhibiting the activated Factor X (FXa) in a selective and competitive manner. DOACs have a relatively short half-life and almost immediate anticoagulant activity, and rapidly reach the plasma peak concentration. Therefore, they do not need a phase of overlapping with parenteral anticoagulants. After their withdrawal, their removal is sufficiently rapid, although influenced by renal function. Dabigatran is the only DOACs to be administered as a pro-drug and becomes active after drug metabolization. The route of elimination of dabigatran is primarily renal, whereas FXa inhibitors are mainly eliminated by the biliary-fecal route. The drug interactions of DOACs are mainly limited to drugs that act on P-glycoprotein for dabigatran and on P-glycoprotein and/or cytochrome P3A4 for anti-Xa. DOACs have no interactions with food. Given their linear pharmacodynamics, with a predictable dose/response relationship and anticoagulant effect, DOACs are administered at a fixed dose and do not require routine laboratory monitoring.
\end{abstract}

\section{Background}

Up to now, venous and arterial thromboembolic diseases have been prevented and treated by using parenteral anticoagulants, such as unfractionated (UFH) or low molecular weight heparins (LMWHs) or fondaparinux and oral anticoagulants acting by inhibiting vitamin K-dependent coagulation factors (vitamin $\mathrm{K}$ antagonists, VKAs). ${ }^{1}$

Unfractionated heparin, LMWHs, fondaparinux and VKAs are indirect anticoagulants.,3 UFH, LMWHs and fondaparinux require the presence of antithrombin (AT) for their anticoagulant activity. ${ }^{2}$ Binding with AT in a selected site of their molecular

Correspondence: Mauro Campanini, AOU Maggiore della Carità, Novara, Italy.

E-mail: mauro.campanini@maggioreosp.novara.it

Key words: new oral anticoagulants, dabigatran, rivaroxaban, apixaban, edoxaban, betrixaban, pharmacokinetics, pharmacodynamics, pharmacology.

Received for publication: 12 April 2013.

Revision received: 4 September 2013.

Accepted for publication: 27 September 2013.

This work is licensed under a Creative Commons Attribution NonCommercial 3.0 License (CC BY-NC 3.0).

CCopyright L. Masotti and M. Campanini, 2013

Licensee PAGEPress, Italy

Italian Journal of Medicine 2013; 7(s8):1-7

doi:10.4081/itjm.2013.s8.1 structure composed by five saccharidic units (pentasaccharide), UFH and LMWHs inhibit active Factor II (thrombin) and active Factor X (FXa) in a different proportion (thrombin/FXa ratio 4:1 for UFH, 1:1 for LMWHs). ${ }^{2}$ Fondaparinux (pentasaccharide) is a synthetic molecule containing only the pentasaccharidic structure of heparins and inhibits solely FXa by binding with $\mathrm{AT} .{ }^{2}$ VKAs inhibit the gamma-carboxylation of vitamin K-dependent coagulation factors (II, VII, IX, X, protein $\mathrm{C}$ and $\mathrm{S}$ ) making them inactive. ${ }^{3}$ Therefore, with the exception of fondaparinux, 'old' anticoagulants act on multiple targets of the coagulation cascade (Figure 1).

Despite their good efficacy and safety profiles in a clinical setting, helping reduce the mortality and morbidity associated with thromboembolic diseases, all parenteral and oral anticoagulants have limitations and these lead to underuse in clinical practice, especially for VKAs. In fact, a very recent meta-analysis confirms that only $60 \%$ of patients suitable for treatment with VKAs effectively receive these drugs. ${ }^{4}$

Briefly, VKAs have an unpredictable pharmacological profile in different patients, based on genetic factors and multiple food and drug interactions. ${ }^{3}$ Therefore, VKAs require close laboratory monitoring of the international normalized ratio (INR) causing patient discomfort and resulting in frequent dose adjustments. Therefore, they have a narrow therapeutic window, the risk of stroke and systemic embolism being increased at lower levels of anticoagulation (INR $<2.0)$ and bleeding risk being higher when INR is over $3.0^{3}$ (Figure 2). VKAs have a long half-life and a slower onset and offset of action requiring a bridging 


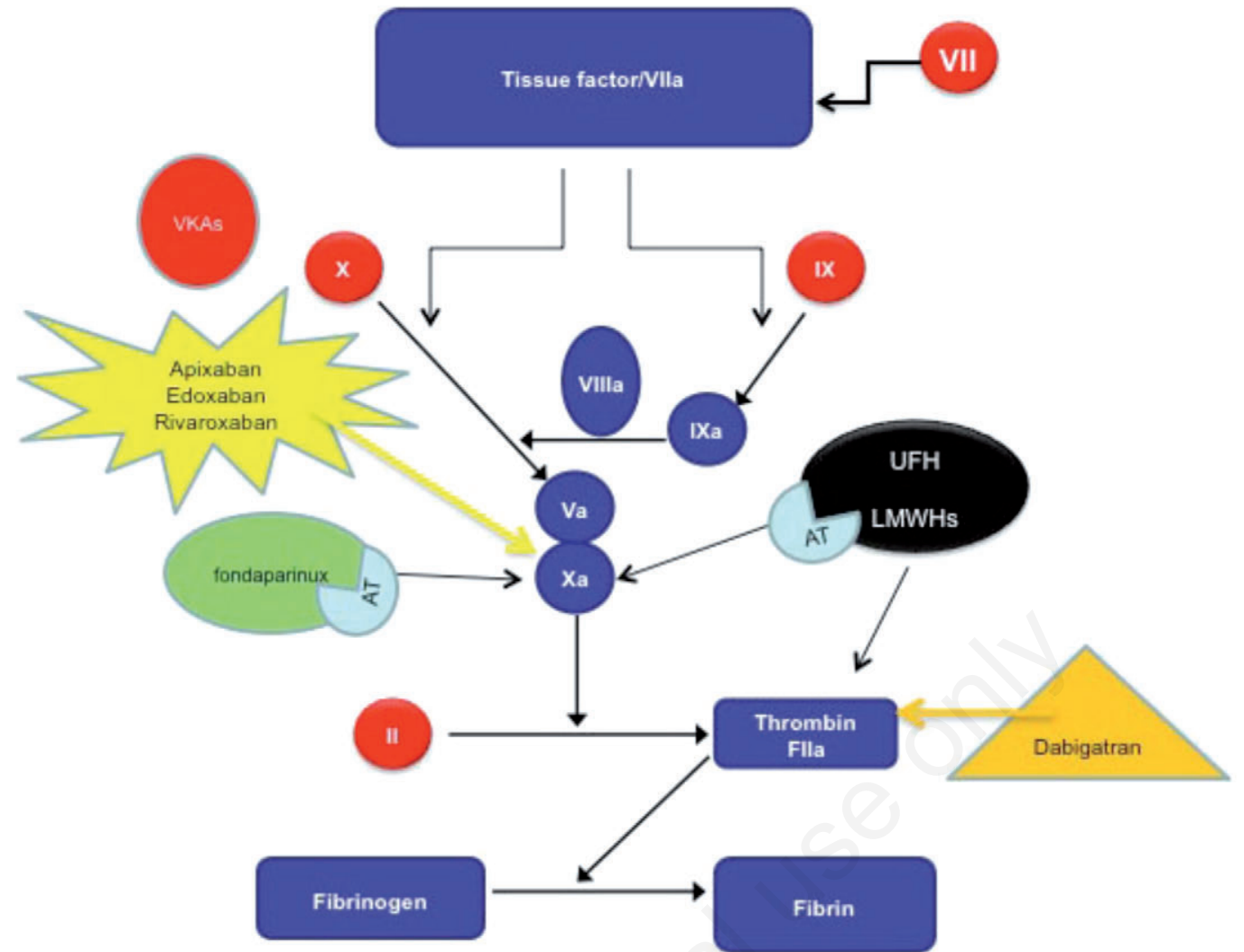

Figure 1. Targets of action of old and new anticoagaulants.

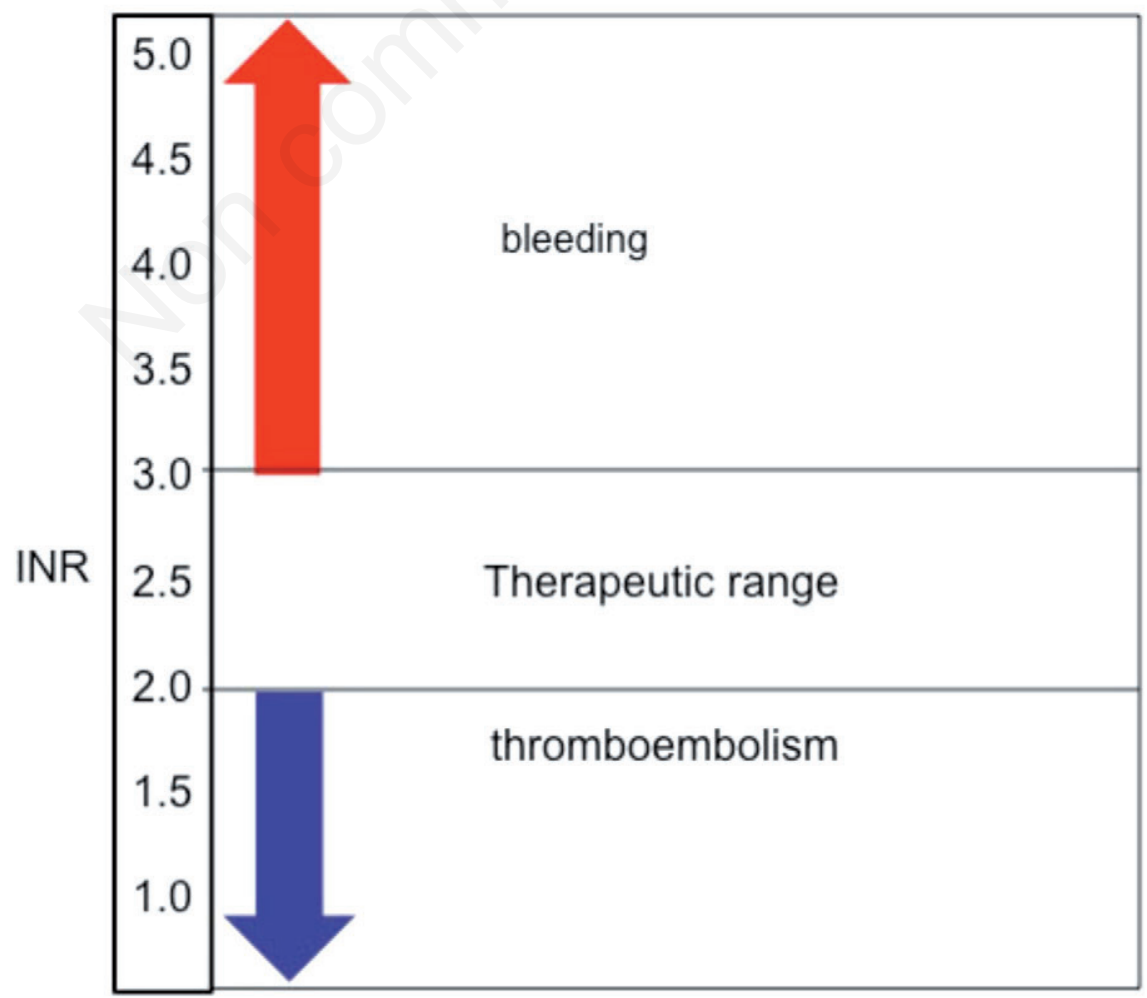

Figure 2. The narrow therapeutic window of vitamin $\mathrm{K}$ antagonists. 
(overlapping) therapy with parenteral anticoagulants such as intravenous (i.v.) or subcutaneous (s.c.) UFH or LMWHs or fondaparinux. After their withdrawal, they are eliminated from plasma in 3-5 days. ${ }^{3}$ Due to these limitations, in real practice, the time in therapeutics range (TTR) is often sub-optimal $(<60 \%) .{ }^{5} \mathrm{VKAs}$ present many contraindications to their use, and these are presented by over $20 \%$ of potential patients. ${ }^{6}$

With both i.v. and s.c. administration, UFH shows a wide variability in its anticoagulant effect due to a lack of linearity in its dose/effect response. Therefore, its anticoagulant effect requires laboratory monitoring by using aPTT, and frequent dose adjustments must be implemented. Its half-life is dose-dependent and its elimination is not influenced by renal function. Druginduced osteoporosis, allergy, and thrombocytopenia are side effects that not negligible. Protamine sulphate is the specific antidote of UFH. ${ }^{2}$

LMWHs are administered s.c. in fixed doses. After administration, these are rapidly absorbed in a linear dose-dependent fashion. Plasma peak concentration is quick and dose-dependent. Bio-availability is approximately $90 \%$. Their anticoagulant activity is more predictable compared to UFH and they do not require laboratory monitoring. Half-life is brief and dose-dependent. Clearance of LMWHs is almost completely influenced by renal function. Drug-induced osteoporosis, allergy and thrombocytopenia could be even less frequent for LMWHs, such as for patients treated with UFH. ${ }^{3}$

Fondaparinux has a fast onset of action, a predictable and dose-dependent anticoagulant activity, and a linear pharmacokinetics. Therefore, it does not require laboratory monitoring. It has a wide bio-availability, a longer half-life compared with LMWHs (approx. $17 \mathrm{~h}$ ) permitting once daily administration. Clearance of fondaparinux is completely through the kidneys. Drug-induced osteoporosis and thrombocytopenia are extremely rare events. ${ }^{3}$

The limitations of VKAs and parenteral anticoagulants have led the pharmaceutical industry to search for new molecules that could overcome these limitations by providing the clinician with more manageable but equally effective and safe drugs. Efforts have also been made to favor, if possible, patient compliance by reducing the discomfort caused by therapy. ${ }^{7}$ Therefore, in recent years, pharmacological research has produced new oral anticoagulant molecules acting on specific and single targets of the coagulation cascade (Figure 1). After the phase I and II studies, new oral anticoagulants (NOAs) have been tested in clinical randomized controlled phase III trials designed to evaluate their efficacy and safety. ${ }^{8}$ NOAs have been defined direct oral anticoagulants (DOACs) to distinguish them from 'old oral anticoagulants' with indirect anticoagulant mechanism of action, as mentioned above. ${ }^{7}$ Those that have been put on the market are divided into two basic groups: the direct thrombin (Factor IIa) inhibitors and the direct inhibitors of activated Factor X (FXa). The suffix-tran identifies the direct thrombin inhibitors while the suffix-xaban identifies the FXa inhibitors. ${ }^{7}$

Due to their brief half-life, rapid achievement of plasma peak concentration and quick onset of action, the DOACs do not require an induction phase in order to determine their anticoagulant effect. Therefore, they do not need an overlapping phase with parenteral anticoagulant drugs. The DOACS have a linear pharmacodynamics with a predictable dose/response relationship and anticoagulant effect. Therefore, they can be administered at a fixed dose and do not require dose adjustment. For the same reason, the DOACs do not require routine laboratory monitoring. ${ }^{7}$

Once the DOACS have been discontinued, their elimination from plasma is relatively fast, especially in patients with normal renal function. The DOACs do not interact with food and have little interaction with other drugs. ${ }^{7}$ All these advantages seem to favor the clinical use of DOACs over VKAs (Table 1).

A detailed description of the mechanism of action and pharmacological properties of the single DOACs with reference to molecules already available for use in clinical practice or soon to be introduced is presented below. It should be stressed that other new direct oral anticoagulants anti-IIa (AZD0837) and anti-Xa (betrixaban, letaxaban, darexaban, eribaxaban, LY517717) are under pre-clinical or dose-finding investigation, and anticoagulant molecules that act by inhibiting Factors VIIa, VIIIa and IXa seem to be ready to enter pharmacological experimentation., ${ }^{7,9}$

\section{Pharmacokinetics}

\section{Oral direct thrombin inhibitors}

Dabigatran is a selective and reversible inhibitor of thrombin. ${ }^{10-15}$ Dabigatran reversibly binds to active site of thrombin both when free and when clot-bound. Dabigatran is administered as pro-drug (dabigatran etexilate). Non-specific enzymes convert the pro-drug to the active molecule (dabigatran). This is of utmost importance because the high polarity of dabigatran means that gastrointestinal absorption is impossible, while the pro-drug can be easily adsorbed after oral intake.

After administration, bioavailability of dabigatran is approximately $6.5 \%$. Time to reach maximum plasma concentration is 1-3 $\mathrm{h}$. Distribution volume is approximately 60-70 liters. Binding with plasma proteins is low (near 35\%). Metabolism starts in the gastrointestinal tract and ends in the liver, but it is not mediated by cytochrome P450. Half-life of dabigatran 
Table 1. Advantages and disadvantages of new and old oral anticoagulants.

\begin{tabular}{lcc}
\hline Characteristics & VKAs & DOACs \\
\hline Mechanism of action & indirect/multi-targets & direct/single-target \\
\hline Onset of action & slow & fast \\
\hline Overlapping with parenteral anticoagulants in the induction phase & yes & no \\
\hline Dose/effect profile & unpredictable & predictable \\
\hline Pharmacodynamics & not linear & linear \\
\hline Dose adjustment & frequent & no \\
\hline Therapeutic window & narrow & wide \\
\hline Food inteference & yes & no \\
\hline Drug interaction & multiple & few \\
\hline Routine laboratory monitoring & necessary & not necessary \\
\hline Half-life & long & brief \\
\hline Elimination & long & brief \\
\hline Antidote & yes & no
\end{tabular}

VKAs, vitamin K antagonists; DOACs, direct oral anticoagulants.

is approximately $8 \mathrm{~h}$ after the first dose and $6 \mathrm{~h}$ after multiple doses. After 4-6 h, the maximum concentration is reduced by approximately $30 \%$. Therefore, dabigatran needs to be administered twice daily. Steady state is reached in three days. Dabigatran is excreted for $80 \%$ from kidneys; therefore, renal failure could result in a reduced excrection, increasing halflife. Dabigatran has no interactions with food but the contemporary intake of food could decrease the plasma peak concentration by $2 \mathrm{~h}$. Drug interferences are limited to drug inhibitors or inductors of P-glycoprotein. Dabigatran has no specific antidote for urgent reversal. Due to low binding with plasma protein, dabigatran is dialyzable. There have been no reports of hepatotoxicity with dabigatran. The drug has not been tested in pregnancy. ${ }^{10-15}$

\section{Oral direct Factor Xa inhibitors}

Rivaroxaban determines a strong inhibition of FXa binding to its active site both when free and when prothrombin (Factor II)-bound. ${ }^{16-20}$ Rivaroxaban has no pro-drug; it has an optimal adsorbent profile through gastrointestinal tract when orally administered. Bioavailability is very high $(80 \%)$. Plasma peak is reached in $2 \mathrm{~h}$. Half-life ranges from $6-8 \mathrm{~h}$ in adults to $12 \mathrm{~h}$ in the elderly. Rivaroxaban is metabolized from liver through a mechanism that is independent of cytochrome P450 but dependent on cytochrome P3A4. Excretion of the active drug is guaranteed from kidneys for $33 \%$, whereas $66 \%$ is due to the fecal-biliary system with interaction with P-glycoprotein. Rivaroxaban does not interact with food, whereas it could have interactions with drugs with action on cytochrome P3A4 and on P-glycoprotein. Due to permanence of a relatively high concentration after $24 \mathrm{~h}$ from oral intake, sufficient for anticoagulant activity, rivaroxaban is administered once daily. Rivaroxaban has no specific antidote for urgent reversal. ${ }^{16-20}$

Apixaban is a selective and reversible inhibitor of FXa. Apixaban acts by binding with the active site of FXa both when free and when thrombin-bound. It is adsorbed through the gastrointestinal tract; it has approximately $50 \%$ bioavailability. Plasma peak is reached after $2 \mathrm{~h}$. Half-time after multiple doses is approximately 9-14 h. Apixaban is metabolized through liver with a mechanism which is dependent on cytochrome P3A4. Excretion is mainly due to the biliary system (75\%), whereas renal excretion is approximately $25 \%$. Apixaban needs to be administered twice daily. Apixaban has no interactions with food, but has interactions with drugs that interact with P-glycoprotein and cytochrome P3A4. Apixaban has no specific antidote for urgent reversal. ${ }^{21-23}$

Edoxaban is a direct inhibitor of Factor Xa of small dimensions. It has been designed from DX9065A, which was one of the first parenteral inhibitors of Factor Xa. Edoxaban has no pro-drug. Edoxaban is rapidly absorbed after a single oral dose and reaches plasma peak concentrations in 1-2 h. Plasma half-life is 6-11 $\mathrm{h}$ after administration of single doses and 9-10 $\mathrm{h}$ after multiple doses. Edoxaban is metabolized by the biliary-fecal system for approximately $65 \%$. Approximately one-third of edoxaban is eliminated by renal excretion. Compared to rivaroxaban and apixaban, 


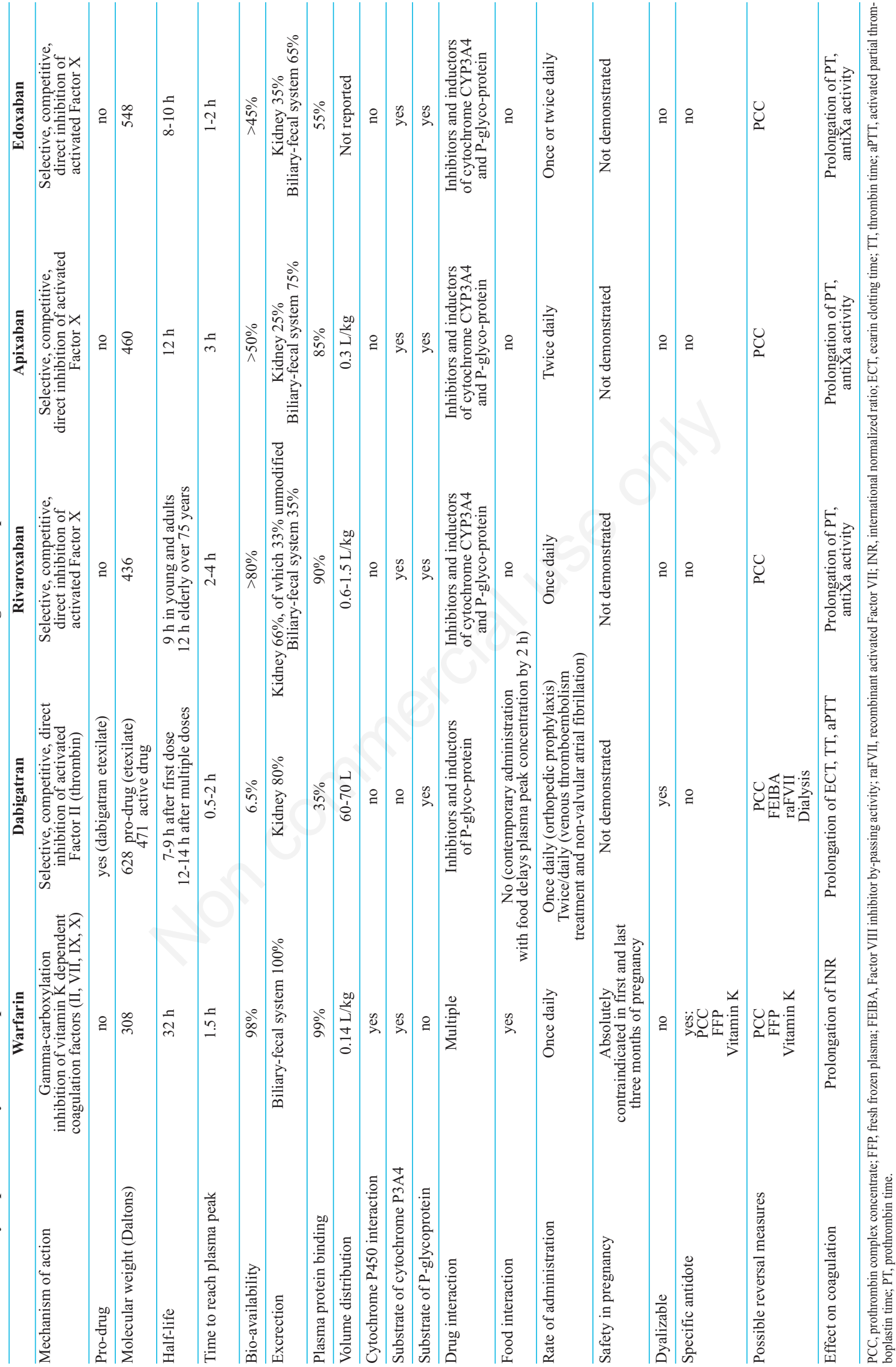


edoxaban has a lower binding capacity of the protein (40-59\%). Like other anti-Xa, edoxaban is a substrate for P-glycoprotein and cytochrome P3A4. Edoxaban has no interactions with food. ${ }^{24-26}$

None of the FXa inhibitors have shown any liver toxicity and none have been tested in pregnancy.

Table 2 shows the main pharmacodynamic and pharmacokinetic characteristics of the new oral anticoagulants compared with warfarin.

\section{Pharmacodynamics}

The DOACs have a linear pharmacodynamics with a predictable dose/response profile. ${ }^{7}$ The plasma concentration and the antithrombotic effect of the DOACs are dose-dependent. At prophylactic or therapeutic doses, DOACs impose modest changes on the common coagulation testing; the effect on coagulation parameters is more evident at the peak of plasma concentration and at steady state. Because of this, the DOACs have been tested in phase III clinical trials without routine laboratory monitoring, which is not recommended in clinical practice. ${ }^{27,28}$ The available data on the effect of DOACs on coagulation parameters have been derived mainly from the pre-clinical, dose-finding studies. ${ }^{28}$

At prophylactic doses and therapeutic doses, dabigatran does not interact substantially on prothrombin time (PT). The activated partial thromboplastin time (aPTT) is prolonged by dabigatran in a curve-linear manner. For lower concentrations, dabigatran prolongs the aPTT in a linear fashion, while at higher concentrations, reached in cases of overdose, the increase in aPTT loses this linearity and tends to plateau. Dabigatran instead prolongs the thrombin time and the ecarin clotting time in a linear, dose and plasma concentration-dependent manner. The inhibitors of Factor Xa determine a prolongation of $\mathrm{PT}$ and a less evident prolongation of aPTT in a concentration-dependent manner, while they result in a linear concentration-dependent increase in the anti-Xa activity. ${ }^{27-30}$

\section{Conclusions}

Direct oral anticoagulants have pharmacokinetic and pharmacodynamic properties that could simplify and, at the same time, increase medical interest in anticoagulation, improve patient compliance and reduce patient discomfort. Considering the good efficacy and safety profiles that have emerged from phase III randomized clinical trials, it seems that we could be near to achieving the ideal anticoagulants. However, some characteristics of DOACs should be carefully taken into account in clinical practice to avoid their incorrect use. These include: i) renal elimination (even though the importance of this differs according to the different molecules used); ii) the interference with drugs interacting with P-glycoprotein and/or cytochrome P3A4; iii) or, finally, the lack of specific antidotes.

\section{References}

1. King CS, Holley AB, Moores LK. Moving toward a more ideal anticoagulant: the oral direct thrombin and factor xa inhibitors. Chest 2013;143:1106-16.

2. Garcia DA, Baglin TP, Weitz JI, et al. Parenteral anticoagulants: Antithrombotic Therapy and Prevention of Thrombosis, 9th ed: American College of Chest Physicians Evidence-Based Clinical Practice Guidelines. Chest 2012;141:e24S-43S.

3. Ageno W, Gallus AS, Wittkowsky A, et al. Oral anticoagulant therapy: Antithrombotic Therapy and Prevention of Thrombosis, 9th ed: American College of Chest Physicians Evidence-Based Clinical Practice Guidelines. Chest 2012;141: e44S-88S.

4. Ogilvie IM, Newton N, Welner SA, et al. Underuse of oral anticoagulants in atrial fibrillation: a systematic review. Am J Med 2010;123:638-45.

5. Baker WL, Cios DA, Sander SD, Coleman CI. Metaanalysis to assess the quality of warfarin control in atrial fibrillation patients in the United States. J Manag Care Pharm 2009; 15:244-52.

6. Kalra L, Perez I, Melbourn A. Risk assessment and anticoagulation for primary stroke prevention in atrial fibrillation. Stroke 1999;30:1218-22.

7. Weitz J, Eikelboom JW, Samama MM. New antithrombotic drugs: Antithrombotic Therapy and Prevention of Thrombosis. 9th ed: American College of Chest Physicians Evidence-Based Clinical Practice Guidelines. Chest 2012; 141:e120S-e151S.

8. Becattini C, Vedovati MC, Agnelli G. Old and new oral anticoagulants for venous thromboembolism and atrial fibrillation: a review of the literature. Thromb Res 2012;129:392-400.

9. Ahrens I, Lip GY, Peter K. New oral anticoagulant drugs in cardiovascular disease. Thromb Haemost 2010;104: 49-60.

10. van Ryn J, Goss A, Hauel N, et al. The discovery of dabigatran etexilate. Front Pharmacol 2013;4:12.

11. Eisert WG, Hauel N, Stangier J, et al. Dabigatran: an oral novel potent reversible nonpeptide inhibitor of thrombin. Arterioscler Thromb Vasc Biol 2010;30:1885-9.

12. Stangier J, Clemens A. Pharmacology, pharmacokinetics, and pharmacodynamics of dabigatran etexilate, an oral direct thrombin inhibitor. Clin Appl Thromb Hemost 2009;15:9S-16S.

13. Blech S, Ebner T, Ludwig-Schwellinger E, et al. The metabolism and disposition of the oral direct thrombin inhibitor, dabigatran, in humans. Drug Metab Dispos 2008;36:386-99.

14. Ufer M. Comparative efficacy and safety of the novel oral anticoagulants dabigatran, rivaroxaban and apixaban in preclinical and clinical development. Thromb Haemost 2010;103:572-85.

15. Squizzato A, Dentali F, Steidl L, Ageno W. New direct thrombin inhibitors. Intern Emerg Med 2009;4:479-84. 
16. Perzborn E, Roehrig S, Straub A, et al. Rivaroxaban: a new oral factor Xa inhibitor. Arterioscler Thromb Vasc Biol 2010;30:376-81.

17. Garcia D, Libby E, Crowther MA. The new oral anticoagulants. Blood 2010;115:15-20.

18. Weinz C, Schwarz T, Kubitza D, et al. Metabolism and excretion of rivaroxaban, an oral, direct factor Xa inhibitor, in rats, dogs, and humans. Drug Metab Dispos 2009;37:1056-64.

19. Abrams PJ, Emerson CR. Rivaroxaban: a novel, oral, direct factor Xa inhibitor. Pharmacotherapy 2009;29: 167-81.

20. Gulseth MP, Michaud J, Nutescu EA. Rivaroxaban: an oral direct inhibitor of factor Xa. Am J Health Syst Pharm 2008;65:1520-9.

21. Raghavan N, Frost CE, Yu Z, et al. Apixaban metabolism and pharmacokinetics after oral administration to humans. Drug Metab Dispos 2009;37:74-81.

22. Nutescu E. Apixaban: a novel oral inhibitor of factor Xa. Am J Health Syst Pharm 2012;69:1113-26.

23. Shantsila E, Lip GY. Apixaban, an oral, direct inhibitor of activated Factor Xa. Curr Opin Investig Drugs 2008; 9:1020-33.

24. Furugohri T, Isobe K, Honda Y, et al. DU-176b, a potent and orally active factor Xa inhibitor: in vitro and in vivo pharmacological profiles. J Thromb Haemost 2008:6: 1542-9.

25. Bathala MS, Masumoto H, Oguma T, et al. Pharmacokinetics, biotransformation, and mass balance of edoxaban, a selective, direct factor Xa inhibitor, in humans. Drug Metab Dispos 2012;40:2250-5.

26. Camm AJ, Bounameaux H. Edoxaban: a new oral direct factor xa inhibitor. Drugs 2011;71:1503-26.

27. Baglin T, Keeling D, Kitchen S. Effects on routine coagulation screens and assessment of anticoagulant intensity in patients taking oral dabigatran or rivaroxaban: guidance from the British Committee for Standards in Haematology. Br J Haematol 2012;159:427-9.

28. Samama MM, Guinet C, Le Flem L. Do new oral anticoagulants require laboratory monitoring? The clinician point of view. Thromb Res 2012;130:S88-9.

29. Tripodi A. The laboratory and the new oral anticoagulants. Clin Chem 2013;59:353-62.

30. Garcia D, Barrett YC, Ramacciotti E, Weitz JI. Laboratory assessment of the anticoagulant effects of the next generation of oral anticoagulants. J Thromb Haemost 2013;11:245-52. 